\title{
Book Review : Surgery of Spinal Cord Tumors Based on Anatomy: An Approach Based on Anatomic Compart- mentalization
}

\author{
Seung Won Park \\ Department of Neurosurgery, Chung-Ang University Hospital, Seoul, Korea
}

To the Editor,

It is a great pleasure to review a book titled 'Surgery of Spinal Cord Tumors Based on Anatomy' which Professor Chun Kee Chung edited (Fig. 1). He should have published the book earlier because he is an expert in spinal tumor surgery and a well-known neurosurgeon and teacher in the world. He also has been leading the Korean Spine Oncology Research Society for more than 10 years. For a long time, many spine surgeons expected and wanted a book to be published showing his experiences and philosophy about the surgical management of spinal tumors. Spinal cord tumor is, in his words, a disease having a potentially devastating impact to the patients themselves as well as the loving persons who shares their lives with the patients. That was the reason why Professor Chung should have been so humble in front of the disease.

The surgeries for spinal cord tumors, unlike other spinal surgeries, are likely to cause damage to normal spinal tissue in the process of removing tumors. However, most books or papers have been described primarily according to the tumor pathologies. Although they wrote the relationships with the surrounding spinal cord tissue according to each tumor pa- thology, they are not surgical approach-oriented descriptions. The tumor pathologies are usually diagnosed with various imaging studies, and, accordingly, the pre-operative diagnosis of spinal cord tumors would not reflect the exact tumor pa-

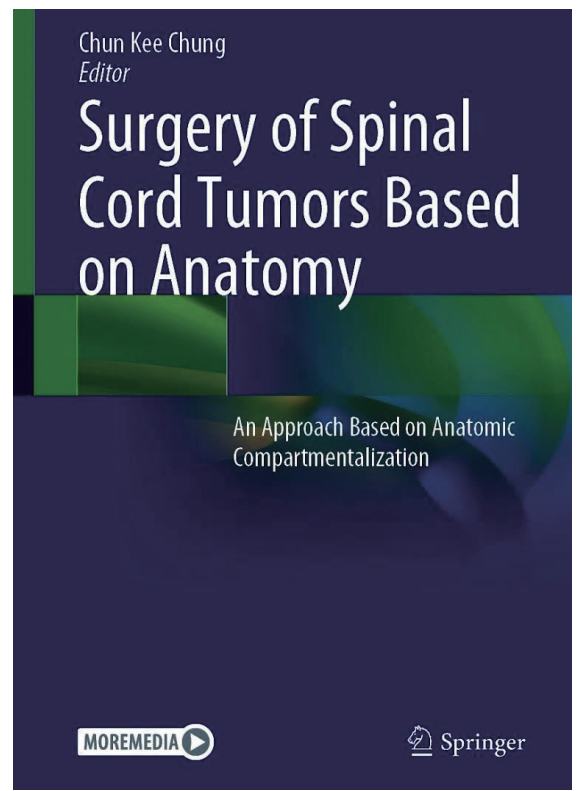

Fig. 1. The book cover.

- Received : April 12, 2021 • Accepted : June 7, 2021

- Address for reprints : Seung Won Park

Department of Neurosurgery, Chung-Ang University Hospital, 102 Heukseok-ro, Dongjak-gu, Seoul 06973, Korea

Tel : +82-2-6299-3190, Fax : +82-2-6299-2069, E-mail : nspsw@cau.ac.kr, ORCID : https://orcid.org/0000-0001-8305-7501

This is an Open Access article distributed under the terms of the Creative Commons Attribution Non-Commercial License (http://creativecommons.org/licenses/by-nc/4.0) which permits unrestricted non-commercial use, distribution, and reproduction in any medium, provided the original work is properly cited. 
thology. Surgeons may meet an unexpected situation different from the histological characteristics of the pre-operatively presumed tumor pathology. A fascinating part of this book is that they identified the exact cause of confusion surgeons experiencing during the spinal cord tumor surgery and tried to solve the problem by classifying the spinal cord tumors by the anatomical compartments in which the tumor is located. The concept of the anatomical compartment can obtain more accurate information of the spinal cord tumor when putting together the pathological and radiological data. More information about the anatomical compartments around the tumor will help the spine surgeons dissect the tumor more safely and confidently in the process of reaching the tumor starting from the spinal cord surface. At the beginning part of the book, they made a chapter for anatomy of spinal meninges and meningeal spaces, which showed a very fresh and sparkling interpretation of the spinal cord structures based on the surgical approach's perspective by an integrated viewpoint of basic anatomy and cell biology major.

In this book, the tumor locations were divided into five extradural-dural compartments and five intradural compartments, and surgical approaches to the compartments were introduced separately. The extradural-dural compartments were classified into extradural nerve plexus, extradural paraspinal, extradural foraminal, extradural intracanal, and dural tumors. These tumors correspond to peripheral nerve tumors. But the dissection and removal of each anatomical barrier can be achieved with minimized damage to normal tissue, understanding that the endoneurium, perineurium, and epineurium around the peripheral nerve are extensions of the pia, arachnoid, and dura of the spinal cord. In foraminal tumors or bone tumors, detailed tips are introduced, including how to deal with the bony structure and the attached nerve tissue to the tumor.

The highlight of a surgical approach based on anatomical compartment is the approach to the intradural compartment. The tumors of this compartment were divided into intradural extra-arachnoidal, sub-arachnoidal, pial-extrapial, intrapial intra-axial, and intra-ependymal tumors. They stressed the importance of arachnoid and pial layers in these compartments. Arachnoid layer is a protective layer for vascular structures underneath it during the removal of extra-arachnoidal tumors such as meningioma. The Arachnoid layer can also be a surgical landmark for exposure of sub-arachnoid tumor surface such as schwannoma. Circumferential coagulation has been recommended for juxtamedullary tumors such as hemangioblastoma, which can cause spinal cord ischemia by damage to the collateral circulations. The risky massive coagulation can be avoided by preserving the pial layer during tumor dissection.

The intra-axial and intrapial intra-ependymal tumors were classified as intramedullary tumors. Usually, posterior midline myelotomy with pial strut suture is helpful to keep the surgical field during removal of intramedullary glioma. Posterolateral myelotomy or direct transpial approach using dorsal root entry zone (DREZ) may be necessary to approach an eccentric intramedullary tumor. Posterolateral myelotomy starts with myelotomy at just medial to the DREZ, which will naturally lead to the posterolateral tract of Lissauer and expose the tumor surface without cord injury. Vascular tumors such as hemangioblastoma or cavernous malformation can be indicated for a direct transpial approach. One of the intriguing parts of this book is the intrapial intra-ependymal tumor, usually ependymoma in the spinal cord. The author speculated that the tumor should be separated as an intradural intraependymal tumor due to its characteristic developmental orientation. They also explained their unique way of tumor dissection following the ependymal layer for safer removal of spinal cord ependymoma.

Of course, the authors did not forget the warning about relying entirely on the surgical approach based on the anatomical compartments. Intraoperative monitoring such as doral column mapping, evoked potential, and electromyography can be used to minimize neural damage during the surgery. Tractography using diffusion tensor images will play a vital role in surgical planning in the future because it provides locational information of tumors and normal nerve fibers threedimensionally.

The anatomical compartment-based approach seems to have a more significant impact on the surgical outcome of the intramedullary tumor. The pre-ganglionic schwannoma is an intramedullary tumor located in the intradural sub-arachnoid space, which can be removed successfully by the anatomical approach. The authors compared their two studies about the surgical results of intrapial spinal cord tumors, which were reported with about 10 years of interval. Unlike the surgeries performed in the previous study, the surgeries performed about 10 years later approached according to the anatomical 
compartment concept. In the case of an astrocytoma, where the anatomical compartment was obscure, there was no difference between the two reports, which suggests limitations of the surgical approach based on the anatomical compartment. However, in ependymoma, where the anatomical compartment was relatively clear, there were fewer recurrences and improved extent of tumor resection, reduced postoperative complications of motor, sensory, and sphincter functions. This book provides surgical video files for various representative cases that help to understand the anatomical compartment concept.

The purpose of the book was to guide or help young surgeons not to make mistakes during surgery of spinal cord tumor. It also seems quite useful for experienced spine surgeons to rethink and readjust many of their procedures. Although Professor Chung emphasized that many of his practices have been changed for a long time, he showed quintessence of spinal cord tumors' management strategies in general and several representative situations, his expensive life-long lessons, in chapter 18. Understanding and taking advantage of the compartmental idea of surgical dissection with the classical principle of a safe approach for spinal cord tumors will improve the degree of surgical removal and reduce surgical problems.

This book is not intended to show up-to-date or cuttingedge technology but share the wisdom learned over a long period of endorsement. It provides a basic understanding of surgical anatomy, which is essential for young spine surgeons in the beginning stages. It also seems to give experienced spine surgeons a chance to understand the causes of various difficult situations during spinal tumor surgery and improve their surgical results. The concept of compartmentalization must be an ultimate weapon for spine surgeons. However, Professor Chung spoke out that new technologies should make further advancements in improving spinal cord tumor management, which will be a heavy responsibility of younger spine surgeons.

\section{CONFLICTS OF INTEREST}

Seung Won Park has been editorial board of JKNS since November 2014. He was not involved in the review process of this original article. No potential conflict of interest relevant to this article was reported.

\section{INFORMED CONSENT}

This type of study does not require informed consent.

\section{AUTHOR CONTRIBUTIONS}

\author{
Writing - original draft : SWP \\ Writing - review \& editing : SWP
}

\section{ORCID}

Seung Won Park https://orcid.org/0000-0001-8305-7501 